\title{
Cerebellar Contributions to Adaptive Control of Saccades in Humans
}

\author{
Minnan Xu-Wilson, ${ }^{1}$ Haiyin Chen-Harris, ${ }^{1}$ David S. Zee, ${ }^{2}$ and Reza Shadmehr ${ }^{1}$ \\ Departments of ${ }^{1}$ Biomedical Engineering and ${ }^{2}$ Neurology, Johns Hopkins School of Medicine, Baltimore, Maryland 21205
}

The cerebellum may monitor motor commands and through internal feedback correct for anticipated errors. Saccades provide a test of this idea because these movements are completed too quickly for sensory feedback to be useful. Earlier, we reported that motor commands that accelerate the eyes toward a constant amplitude target showed variability. Here, we demonstrate that this variability is not random noise, but is due to the cognitive state of the subject. Healthy people showed within-saccade compensation for this variability with commands that arrived later in the same saccade. However, in people with cerebellar damage, the same variability resulted in dysmetria. This ability to correct for variability in the motor commands that initiated a saccade was a predictor of each subject's ability to learn from endpoint errors. In a paradigm in which a target on the horizontal meridian jumped vertically during the saccade (resulting in an endpoint error), the adaptive response exhibited two timescales: a fast timescale that learned quickly from endpoint error but had poor retention, and a slow timescale that learned slowly but had strong retention. With cortical cerebellar damage, the fast timescale of adaptation was effectively absent, but the slow timescale was less impaired. Therefore, the cerebellum corrects for variability in the motor commands that initiate saccades within the same movement via an adaptive response that not only exhibits strong sensitivity to previous endpoint errors, but also rapid forgetting.

\section{Introduction}

It is thought that saccades are highly practiced, optimized examples of ballistic movements. Yet, the motor commands that initiate an eye movement to a given target are variable, and this variability is not random noise. For example, people make saccades with higher velocities in anticipation of seeing a more interesting visual stimulus (Xu-Wilson et al., 2009). Repeating a visual target or reducing the reward associated with a target reduces saccade velocities (Straube et al., 1997; Chen-Harris et al., 2008). On the other hand, increasing the reward associated with the target or making the target the goal of both the eye and the arm movements increases saccade velocities (van Donkelaar, 1997; Snyder et al., 2002; Takikawa et al., 2002). Despite this variability in the motor commands that initiate saccades, the brain accurately guides the eyes to the target. How is this accomplished?

Perhaps endpoint accuracy is possible because the brain incorporates an internal feedback process that monitors the motor commands and corrects them online (Robinson, 1975). If the internal feedback is intact, variability in the commands that initiate the saccade might be compensated via commands that arrive later during the same saccade. However, for this internal feedback

Received July 1, 2009; revised Aug. 25, 2009; accepted Aug. 29, 2009.

This work was supported by grants from the National Institutes of Health (NS37422, EY19581, and EY01849). Minnan Xu-Wilson and Haiyin Chen-Harris were both supported by National Research Service Award predoctoral fellowships from the National Institute of Neurological Disorders and Stroke. We thank Dale Roberts and Adrian Lasker for their superb technical support. We thank the reviewers who provided thorough and insightful comments that greatly improved this manuscript.

Correspondence should be addressed to Minnan Xu, Johns Hopkins School of Medicine, 416 Traylor Building, 720 Rutland Avenue, Baltimore, MD 21205. E-mail: mxu@bme.jhu.edu.

D01:10.1523/JNEUROSCI.3115-09.2009

Copyright $\odot 2009$ Society for Neuroscience $\quad$ 0270-6474/09/2912930-10\$15.00/0 to be effective, it needs to be adaptive and learn from endpoint errors. A computational framework that captures these ideas is one in which motor commands are monitored via a forward model, predicting sensory consequences and allowing for withinsaccade compensation. Endpoint errors should produce adaptation in the forward model because they reflect a prediction error. If there is such an internal feedback process, damage to it might produce both an inability to compensate for the variability in the motor commands that initiated the movement, and an inability to learn from endpoint errors. That is, the two abilities should be correlated.

Previously, we observed that when a visual target on the horizontal meridian was moved vertically as a saccade was made toward it (cross-axis paradigm), saccades became curved (ChenHarris et al., 2008). This suggested that the forward model was learning from endpoint errors, steering the saccade to the target. Here, we looked for the neural basis of this hypothetical internal feedback process by examining saccades of cerebellar patients, as their saccades are dysmetric, and the cerebellum has long been hypothesized to function as an internal feedback pathway that "steers" the eyes to the target (Zee et al., 1976; Optican and Robinson, 1980; Quaia et al., 1999).

Our subjects were a group of patients who suffered from spinocerebellar ataxia type 6 (SCA-6), a neuro-degenerative disease that targets the Purkinje cells of the cerebellum (Sasaki et al., 1998; Honjo et al., 2004; Koeppen, 2005). Our experiment (the cross-axis paradigm) involved repetition of a visual target, something that we had found to produce structured variability in the motor commands that initiate the saccade (Chen-Harris et al., 2008). We wondered whether this natural variability would also be present in the motor commands that initiated saccades of 
A

\begin{tabular}{|c|c|c|c|c|}
\hline Oblique & error-clamp I & Adaptation & De-adaptation & error-clamp II \\
\hline 112 trials & 60 trials & 500 trials & 80 trials & 140 trials \\
\hline 1 & 1 & $(7.5,5)$ & 1 & 1 \\
\hline & $(7.5,0)$ & 1 & $(-7.5,0)$ & $(7.5,0)$ \\
\hline i & 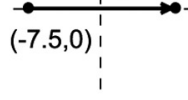 & $\begin{aligned} \\
(-7.5,0) \\
\vdots\end{aligned}$ & $\left.\right|_{1} ^{-}$ & $(-7.5,0) !$ \\
\hline
\end{tabular}

B Adaptation trial

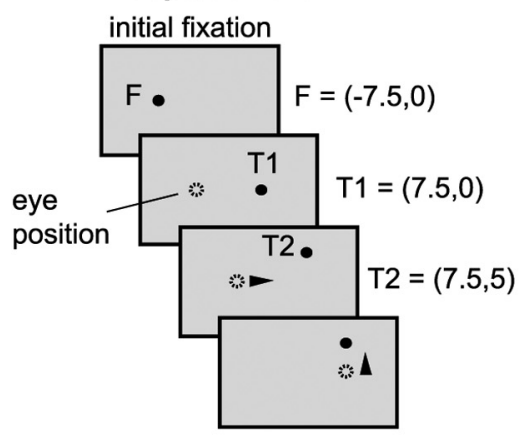

C Error Clamp Trial

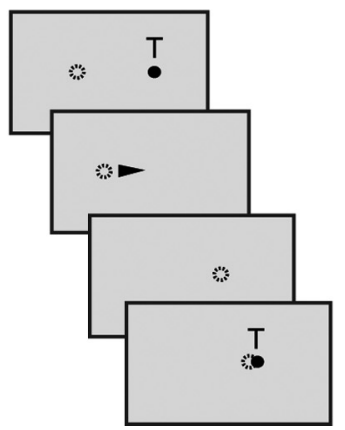

Figure 1. Experimental procedures. $A$, Subjects were trained on a cross-axis adaptation task. The experiment consisted of five blocks: trials in which saccade targets were presented at various oblique angles, 60 preadapt error-clamp trials in which targets were always at $15^{\circ}$ horizontal (error-clamp I), 500 adaptation trials (target jump is counter-clockwise), 80 deadaptation trials (target jump is clockwise), and 140 postadapt error-clamp trials (error-clamp II, targets again at $15^{\circ}$ horizontal). During errorclamp trials, the target did not jump but disappeared after saccade onset and reappeared $500 \mathrm{~ms}$ later at the current eye position. The dashed lines indicate axes centered straight ahead. $\boldsymbol{B}$, Adaptation trials. Filled circles indicate current laser position. Arrowheads indicate when a saccade began. A target was projected $15^{\circ}$ away from fixation (T1). As soon as the saccade began, the target jumped $5^{\circ}$ vertically to a new location (T2). The jump direction was consistently counterclockwise to the orientation of $\mathrm{T1}$. T2 then served as the fixation point (F) for the next trial. In deadaptation trials, the jump direction was clockwise to the orientation of T1. C, Error clamp trials. T1 was presented at $15^{\circ}$ to the left or right of fixation. Once the saccade began, T1 disappeared. Five-hundred milliseconds later, a fixation point appeared with a horizontal position the same as $\mathrm{T} 1$ and a vertical position of the eye from $10 \mathrm{~ms}$ prior.

cerebellar patients, and if so, whether these subjects would show a reduced ability to compensate for that variability.

A second part of our experiment was the endpoint errors that were caused by jumping of the target. We had earlier found that the adaptive response to errors appeared to involve two or more timescales: a fast timescale that learned a great deal from error but had poor retention, and a slower timescale that learned little from error but had good retention (Ethier et al., 2008). Cerebellar damage is known to profoundly impair the ability to adaptively control saccades (Straube et al., 2001; Golla et al., 2008). Here, we wondered whether these two timescales were uniformly affected by cortical cerebellar damage, or was there a greater impairment in one timescale of the adaptive process than in the other.

\section{Materials and Methods}

Experimental setup and design. Subjects sat in a dark room with their head restrained using a dental bite-bar. We used a scleral search coil system (Skalar Medical) to record horizontal and vertical eye movements from either the right or the left eye (Robinson, 1963). Raw eye position information from the coils was filtered in hardware $(90 \mathrm{~Hz}$ low-pass Butterworth), digitized $(1000 \mathrm{~Hz})$, and saved on computer for later analysis. A $0.2^{\circ}$ red laser beam was rear-projected onto a translucent screen located $1 \mathrm{~m}$ in front of the subject. Target position was varied by a galvo-controlled mirror.

The experiment is summarized in Figure 1A. It consisted of 5 blocks: preadaptation oblique (112 trials), preadaptation error-clamp (error-clamp I, 60 trials), counterclockwise (CCW) cross-axis adaptation (500 trials), clockwise (CW) deadaptation trials (80 trials), and postadaptation errorclamp trials (error-clamp II, 140 trials). The experimental blocks are ex- plained in detail below. Each block was further divided into short sets of $\sim 60$ trials ( $1.5 \mathrm{~s}$ intertrial interval). On each trial, the saccade crossed the vertical meridian. The sets were separated with a break typically of $30 \mathrm{~s}$. During set breaks the subjects sat quietly, usually with their eyes closed, except for occasional breaks that were 2-3 min long to administer eye drops to the subject.

Oblique trials. The experiment began with two sets of oblique trials (Fig. $1 A$ ). The target locations were always $15^{\circ}$ away from fixation in the horizontal direction and $0,1,2$, or $3^{\circ}$ above or below the meridian vertically. The targets appeared randomly within the set. Saccades alternated rightward and leftward symmetric about the midline.

Error-clamp trials. The target T1 was presented at $15^{\circ}$ to the left or right of fixation. Once the saccade began, T1 disappeared. At $500 \mathrm{~ms}$ later, a fixation point appeared with a horizontal position the same as that of $\mathrm{T} 1$ and a vertical position of the eye from $10 \mathrm{~ms}$ prior. Therefore, in error-clamp trials we attempt to assay the motor output after adaptation while preventing further learning by minimizing the endpoint errors. Because in the cross-axis paradigm, learning takes place in the vertical direction, the error-clamp trials "clamp" the vertical error to zero but do not affect the horizontal error. To prevent accumulation of a large vertical offset, we restricted the target position to within $\pm 10^{\circ}$ range vertically. Once outside of this range, the vertical position of the target was reset to $0^{\circ}$ for the next trial. There were two error-clamp sets of trials, one before the adaptation sets (error-clamp I) and one after (error-clamp II).

Cross-axis trials. The pattern of target positions is shown in Figure $1 \mathrm{~A}$. A target was projected at $15^{\circ}$ with respect to fixation (T1). As soon as the saccade began, the target jumped $5^{\circ}$ vertically to a new location (T2). In the adaptation trials, the jump direction was consistently counterclockwise to the orientation of T1. T2 then served as the fixation point (F) for the next trial. In deadaptation trials, the jump direction was clockwise to the orientation of T1. The primary saccade to T1 was always followed by a secondary "corrective" saccade that brought the eyes to T2. The data presented here represents characteristics of only the primary saccade.

The transition between CCW to CW adaptation trials occurred midset without a break: the ninth adaptation set began with 20 CCW trials but then suddenly changed to CW training (40 trials). Similarly, the transition from CW to error-clamp was mid-set without a break: the 10th adaptation set began with $40 \mathrm{CW}$ trials but then suddenly changed to error-clamp trials (20 trials).

Subjects. Nine individuals with cerebellar degeneration participated in the study (Table 1). All but one were diagnosed with SCA- 6 , a neurodegenerative disease that primarily affects the Purkinje cells of the cerebellum, particularly in the vermis (Sasaki et al., 1998; Honjo et al., 2004). Magnetic resonance imaging scans confirmed that these patients had global degeneration of the cerebellum including the vermis and the hemispheres. The one patient without a genetic diagnosis (P2) had a clinical picture indistinguishable from the others with a genetically confirmed diagnosis. SCA- 6 is caused by mutations in a gene that encodes a calcium channel in Purkinje cells. In the cell culture models of this mutation, the result is premature death of the Purkinje cells. In the surviving cells, excitability is decreased. Postmortem examination of the brain shows a severe loss of Purkinje cells, with very mild loss of granule, stellate, and basket cells, as well as little or no loss of cells in the inferior olive (Ishikawa et al., 1999). The mutation is a trinucleotide repeat of CAG, with longer repeats resulting in symptoms beginning at an 
Table 1. Cerebellar patients

\begin{tabular}{llllc}
\hline Patient & Age & Gender & Ataxia type & Disease duration (years) \\
\hline P1 & 45 & F & SCA-6 & 7 \\
P2 & 35 & F & SCA, type unknown & 13 \\
P3 & 53 & F & SCA-6 & 11 \\
P4 & 55 & F & SCA-6 & 6 \\
P5 & 74 & M & SCA-6 & 35 \\
P6 & 59 & F & SCA-6 & 14 \\
P7 & 63 & M & SCA-6 & 12 \\
P8 & 67 & F & SCA-6 & 15 \\
P9 & 64 & F & SCA-6 & 4 \\
\hline
\end{tabular}

Seven patients are female; mean age 57 years, range $35-74$ years. F, Female; $M$, male.

earlier age (Matsuyama et al., 1997). Table 1 summarizes the patient information.

Control 1. Eleven age matched control subjects also took part in our experiment (six female and five male; mean age 57 years, range 45-69). All subjects gave written consent to protocols approved by the Johns Hopkins Institution Review Board.

Control 2. In our age-matched control group 1 we noted a trend in which the ability to control for horizontal variability correlated with the ability to learn in the cross-axis adaptation paradigm. To improve our power to detect such a pattern, we enlarged our control group by adding six non-age-matched control subjects (age range $20-43$ years). This younger group allowed us to validate the previous correlations that we had seen in Control 1. The authors M.X. and R.S. were part of the Control 2 group.

Data analysis. The beginning and end of saccades were determined by a $16 \%$ speed threshold. Criteria for including saccades in analysis were as follows: (1) Saccade amplitude must be $>50 \%$ of the target displacement. (2) Saccade duration must be within 50-150 ms. (3) Saccade reaction time must lie within 100-500 ms. (4) Peak horizontal velocity must be $>100 \%$ s. On average, control subjects had $13 \%$ of their saccades excluded while patients, with their more variable saccades, had $26 \%$ excluded.

We use a constant bin width (bw) of 4 to show the effect of stimulus repetition and learning that evolves over the entire course of the experiment (see Figs. $4 A, D, 5 A, 6 A$ ). We then used a smaller bin width of 2 to show the rapid changes that take place at set breaks and sudden changes in trial-type (see Figs. $4 B, C, E, F, 5 C, D, 6 C, D$ ).

The amount of adaptation was assessed by comparing (using a paired, 2-tailed $t$ test) the vertical eye movement averaged over all the baseline error-clamp trials with the vertical movement averaged over the last 60 -trial set of the CCW cross-axis adaptation block. Likewise, the recovery of the previous adaptation that occurred following the brief deadaptation block was assessed by comparing the vertical movement in the baseline trials with the vertical movement averaged over the first set of postadaptation error clamp trials. We used a significance level of $p<0.05$ for each pairwise comparison. We used a $2 \times 1$ repeated-measure ANOVA design to compare the effect of group (control and cerebellar) and the within-subject factor of set number on saccade horizontal parameters (peak horizontal velocity and amplitude). Significance level was set at $p<0.05$. Two-sided unpaired $t$ tests were used to assess the differences in adaptation and recovery levels between groups.

The vertical bias (in Fig. 7C) was calculated as the difference between the absolute of the saccade's vertical endpoint and the absolute of the target's vertical position. For example, a vertical endpoint of $-2.5^{\circ}$ to a target with vertical eccentricity of $-3^{\circ}$ had a bias of $-0.5^{\circ}$, which is undershooting.

The SE of various parameters (Fig. 6, error bars) were calculated as follows. The SE of the adaptation amount $s$ is approximated as $s / \sqrt{n}$, where $n$ is the number of trials used to calculate this measure. The SEs of horizontal and vertical variability as measured by SD $\sigma$ are approximated as $0.71 \sigma / \sqrt{\mathrm{n}}$.

\section{Results}

We will first focus on the horizontal component of eye movements to illustrate that there is variability in the motor com-
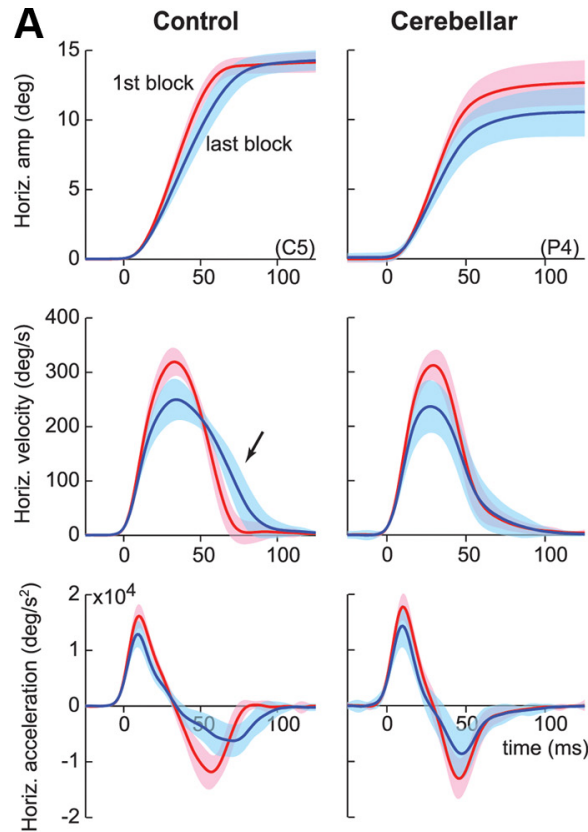

B

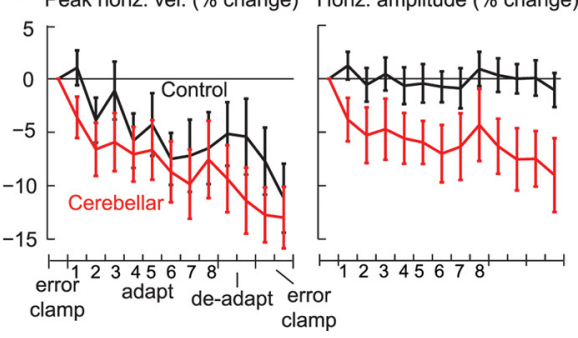

Figure 2. Cerebellar patients could not correct for variability in the motor commands that initiated saccades. $\boldsymbol{A}$, The average horizontal amplitude, velocity, and acceleration traces from the first and last error-clamp blocks (error clamp I and II), in response to a target at $15^{\circ}$, for two representative subjects. In the last block, the saccades of both the control subject (C5) and the cerebellar patient (P4) were initiated with reduced velocities, but the control subject compensated later during the same saccade. Shading indicates SD. B, Group data for horizontal peak velocity and amplitude changes. Percentage change is with respect to the first error-clamp block (error clamp I). Each point is the average from one set of 60 trials. Error bars indicate SEM.

mands that initiate saccades, and that the cerebellum plays a role in the within-saccade compensation for this variability. We will then focus on the vertical direction and the process of adaptation.

\section{A role for the cerebellum in compensating for the variability in the motor commands that initiate saccades}

Figure $2 \mathrm{~A}$ shows the horizontal component of the saccade's kinematics from the first and last error-clamp sets for a typical healthy control (C5) and a representative cerebellar patient (P4). In the control subject, the amplitude of the movement was the same in the first and last sets $(p=0.34)$. However, the amplitude dropped by $2.2^{\circ}$ for the cerebellar patient $(p<0.001)$. The peak velocity dropped in both subjects from the first to the last set: the control subject's saccades slowed by $68.3 \%$ or $21 \%(p<0.001)$ and the cerebellar patient's saccades slowed by $68 \%$ or $24 \%(p<$ $0.001)$. In the control subject, the saccades in the last set started with reduced peak velocities but then were completed with an increased velocity late in the same saccade (see arrow). In the cerebellar patient, the reduction in peak velocity was left uncompensated. Saccade peak accelerations showed similar drops for both subjects: $20 \%(p<0.001)$ for the control subject and $18 \%$ 


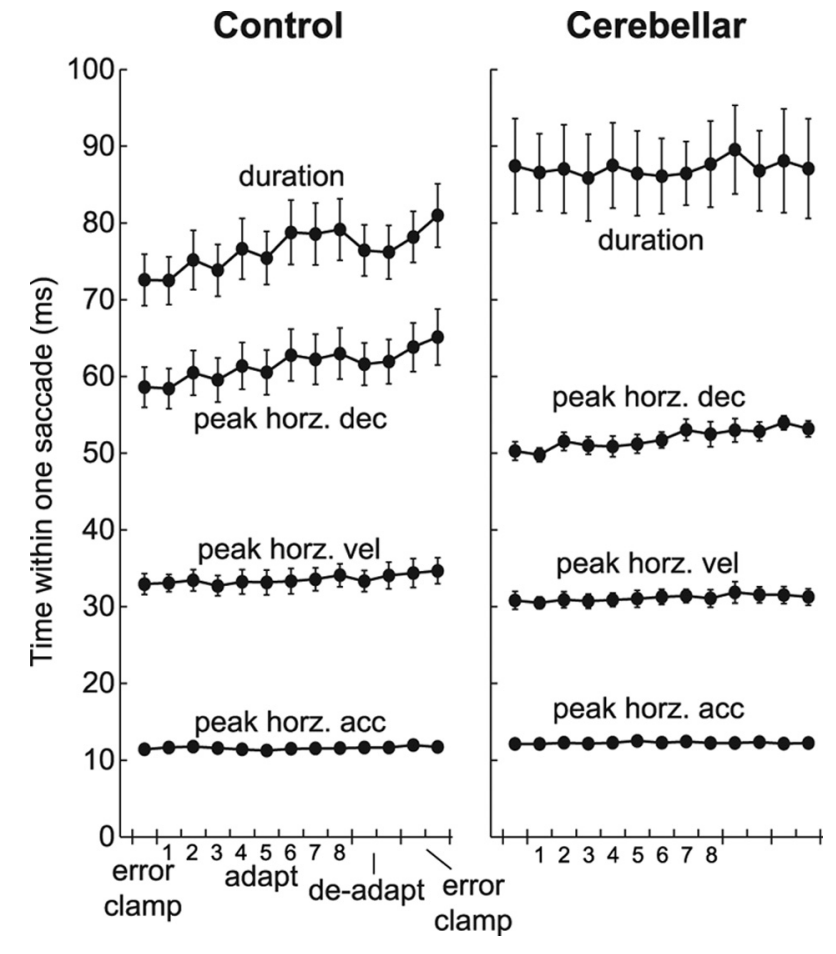

Figure 3. Saccades of control and cerebellar subjects differed most in the deceleration phase. The plots show the changes in the timing of saccade parameters. Position, velocity, and acceleration refer to horizontal components of the movement. Each point is the average from one set of 60 trials. Error bars indicate SEM.

$(p<0.001)$ for the cerebellar patient. The deceleration pattern, however, was strikingly different. The peak value of deceleration of the control subject was less and occurred later than the cerebellar patient. Therefore, from the first to the last set, the magnitude of the motor commands that initiated saccades dropped for both subjects, but only the control subject was able to maintain amplitude by compensating late in the saccade's time course.

Figure $2 B$ summarizes some of these changes in the horizontal direction through the course of the experiment. Both groups made slower saccades as the experiment proceeded. In control subjects, saccades slowed by $11.1 \%$ or $33 \%$ s $(p<0.005)$. In cerebellar patients, saccades slowed by $12.9 \%$ or $40 \%$ s $(p<0.01)$. Repeated measure ANOVA on peak velocity showed an effect of set $(p<0.001)$, no effect of group, and no interaction between group and set. Therefore, saccades slowed by a similar amount in the two groups. Repeated measure ANOVA of horizontal amplitude showed an effect of set $(p<0.01)$, group $(p<0.05)$, and group by set interaction $(p<0.05)$. Control subjects were able to maintain endpoint amplitude from first to last set $(p=0.43)$, while cerebellar patients showed a drop in amplitude by $9.0 \%$ or $1.06^{\circ}(p<0.05)$.

An analysis of saccade timing parameters demonstrated that control and cerebellar subjects differed most in the deceleration phase of saccades (Fig. 3). The time of peak acceleration remained unchanged in both groups ( $p=0.45$ for controls and $p=0.59$ for patients). In the control group, the time of peak velocity ( shift $=$ $1.7 \mathrm{~ms}, p<0.01$ ) and time of peak deceleration ( $p<0.01)$ shifted to a later time, and duration of the saccade (shift $=8.4 \mathrm{~ms}, p<0.001$ ) increased. For cerebellar patients, however, time of peak deceleration showed less than half the change seen in controls ( shift $=2.9 \mathrm{~ms}, p<0.01$ ) while time of peak velocity and duration of saccades showed no significant changes. As a result, while in both groups the commands that accelerated the eyes along the horizontal dimension decreased from the first to the last set of the experiment, the healthy subjects were able to maintain horizontal amplitude by compensating later in the saccade.

In the above data we averaged saccade parameters in each set, and then displayed the results across sets. However, there were also consistent changes that occurred within each set. Each set consisted of 60 trials (intertrial interval of $\sim 1.5 \mathrm{~s}$ ). Between the sets our subjects rested for $\sim 30 \mathrm{~s}$ and closed their eyes. As in our previous studies of cross-axis adaptation (Chen-Harris et al., 2008), on-axis adaptation (Ethier et al., 2008), or simply control studies in which targets did not jump (Chen-Harris et al., 2008), we found that the peak horizontal velocity dropped within each set and then sharply increased in the first saccade after the set break (Fig. 4A). On average, the healthy subjects showed $49.8^{\circ} \mathrm{s}$ or $16.3 \%$ increase in peak horizontal velocity (last two saccades before set break vs first two saccades after set break, $p<0.001$ ) and $10.0 \mathrm{~ms}$ or $15.4 \%$ decrease in duration $(p<0.001)$, as shown in Figure $4 B$. The set breaks also produced a small increase in the horizontal amplitude $\left(0.6^{\circ}\right.$ or $\left.4.6 \%, p<0.05\right)$ (supplemental Fig. 1 , available at www.jneurosci.org as supplemental material) and a small decrease in saccade latencies $(p<0.05)$ (supplemental Fig. 1 , available at www.jneurosci.org as supplemental material). The small but significant increase in amplitude at set breaks suggests that even in healthy people, some of the variability in motor commands that accelerated the eyes was left uncompensated. This is a crucial finding for us as we will later show that the amount of within-saccade compensation in the horizontal direction is a predictor of the ability of that subject to learn from endpoint errors in the vertical direction.

Similar to control subjects, cerebellar patients showed an obvious structure in their saccade velocities: set breaks induced an increase of $28.0 \%$ or $9.3 \%$ in peak horizontal velocity $(p<0.01)$ (Fig. $4 E$ ). This was a clear finding, as set breaks induced an increase in peak horizontal velocity in 7 of 9 cerebellar subjects $(5$ subjects with $p<0.05$ and 2 subjects with $p<0.1$ ), and in 7 of 9 set breaks (supplemental data and supplemental Fig. S2, available at www.jneurosci.org). However, unlike controls, the cerebellar patients did not decrease the saccade durations to compensate for this increased velocity $(p=0.92)$.

In summary, motor commands that accelerated the eyes along the horizontal dimension were affected by two forms of variability: set breaks produced a sharp increase, while target repetition produced a gradual decrease. In healthy people, this variability appeared to be corrected within the same saccade, whereas in the cerebellar subjects, the variability produced dysmetria.

\section{What caused the variability in the motor commands that initiated the saccades?}

It is possible that the experiment induced use-dependent fatigue in the neuronal or muscular structures of the oculomotor system, and the set breaks allowed recovery from this fatigue. However, data from a crucial component of our experiment argued against this possibility: after 20 trials in the final adaptation set, the target sequence unexpectedly switched from a counter-clockwise to a clockwise sequence (start of deadaptation, first red line in Fig. $4 A$ ). If fatigue is a form of habituation in the sensory neurons that convey target information to the motor system, then the transition from adaptation to deadaptation should not produce a recovery because the stimuli that elicited horizontal saccades activated precisely the same retinal location as before. Similarly, if fatigue is a form of use-dependent reduction in the response of 
the extra-ocular muscles, then there should be no recovery because the transition from adaptation to deadaptation did not include a rest period. However, if the fatigue is due to a top-down factor, for example, a decline in an attentional state, then the surprising event should produce recovery.

The unexpected change in the position of a vertical target produced an immediate and robust recovery of velocities in response to the subsequent horizontal target. In Figure 4, $C$ and $F$, the saccade parameters are aligned to the first trial after the target sequence changed from counterclockwise to clockwise target jumps. Changes in percentage are calculated with respect to the trial before the sequence change. In controls, we found an increased velocity of $32.9 \%$ or $11.8 \%$ $(p<0.05)$ and decreased duration of 12.5 ms or $16.9 \%(p<0.01)$. Horizontal displacement $(p=0.64)$ and reaction time $(p=0.20)$ did not change significantly (supplemental Fig. $1 C$, available at www. jneurosci.org as supplemental material). Importantly, this recovery was smaller the second time the target sequence changed (at the end of the deadaptation block, second red line in Fig. $4 \mathrm{~A}$ ): controls showed a $20.4 \%$ or $7.7 \%$ increase in peak velocity $(p=0.15)$. That is, the change in the target sequence produced a sharp increase in velocities when it first occurred, but a smaller one when it repeated.

In cerebellar patients, the unexpected change in target sequence produced $24.9 \%$ or $7.3 \%$ increase in peak velocities, but these changes did not reach significance $(p=0.22)$. There were no significant changes in duration $(p=0.57)$, horizontal amplitude $(p=$ $0.51)$, or reaction time $(p=0.53)$. Therefore, the data from the control subjects suggested that the changes in the commands that initiated the saccades were probably not due to a neuronal or muscular fatigue process that required passage of time for recovery. Rather, the fact that an unexpected change in the stimulus restored peak velocities suggests that the decline in velocity was due to reduced motivation, boredom, or a similar top-down effect.

\section{Adaptation and the multiple timescales of memory}

Our experiment was designed to not only quantify adaptation capabilities of healthy and cerebellar patients, but to potentially unmask the multiple timescales that underlie this adaptation. We considered a common paradigm in which a long period of adaptation was followed by a brief period of deadaptation (Kojima et al., 2004; Criscimagna-Hemminger and Shadmehr, 2008; Ethier et al., 2008). If there are multiple timescales that support adaptation, then behavior should exhibit signatures of these timescales (Smith et al., 2006; Kording et al., 2007). For example, a fast timescale of adaptation should produce forgetting with passage of time (during set breaks), and forgetting with removal of the adaptationdriving errors (during error-clamp trials). This fast timescale should also produce rapid learning in the presence of error. A
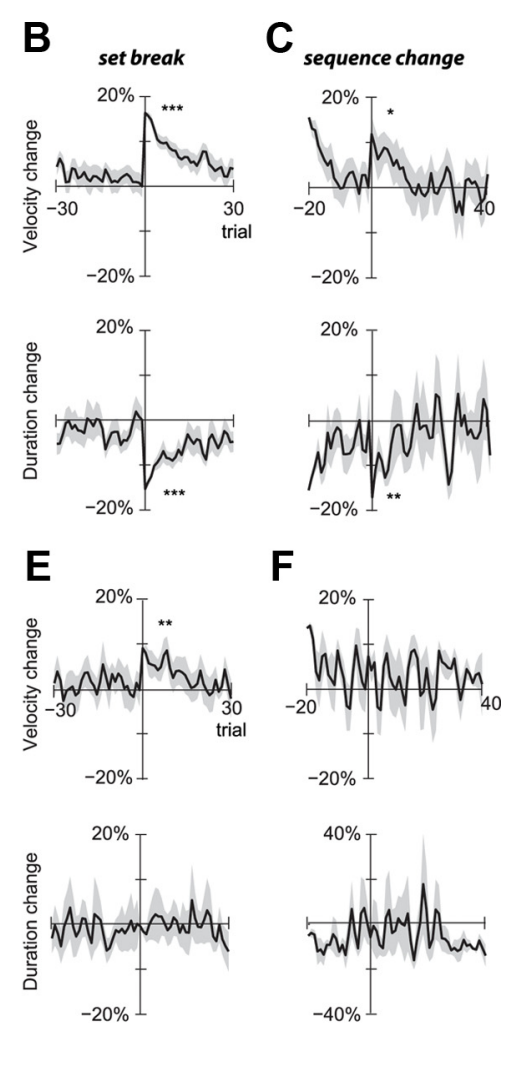

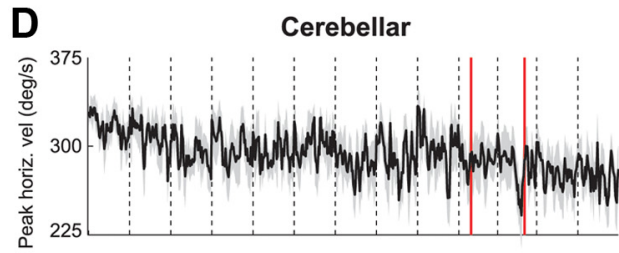

Figure 4. Effect of set breaks on saccade horizontal velocities and durations. $\boldsymbol{A}, \boldsymbol{D}$, Peak vertical velocity and duration, averaged group. Dotted vertical lines mark set boundaries. Each set consisted of 60 trials. Red lines mark sudden changes in target $\boldsymbol{C}, \boldsymbol{F}$, Within-subject changes in peak velocity and duration, aligned to the sudden change in sequence of targets from CCW to $C W$ cross-axis target jumps. $\boldsymbol{A}-\boldsymbol{C}$ are control data, and $\boldsymbol{D}-\boldsymbol{F}$ are cerebellar data.

slow timescale of adaptation should resist deadaptation (when errors reverse direction, termed "extinction") and produce spontaneous recovery toward the previously adapted state in the postadaptation error-clamp period. In the cerebellar patients, is the damage predominantly affecting one timescale of adaptation?

During the adaptation block, as the saccade was initiated the target at the horizontal meridian jumped vertically (in a counterclockwise direction), resulting in an endpoint error which was corrected with a second saccade. In response to this endpoint error, both the control and the cerebellar groups learned to produce primary saccades that had increasing vertical motor commands, but the learning was significantly smaller in the cerebellar patients (Fig. 5A). For example, the change in vertical endpoint from the preadapt error-clamp trials to the last set of adaptation was $+2.01^{\circ}$ in control subjects $\left(p<10^{-4}\right)$, and $+0.56^{\circ}$ in cerebellar patients $(p<0.05)$, with the change being significantly smaller in the cerebellar patients (Fig. $5 B$ ).

Despite the significant amount of adaptation in the saccades made by the cerebellar patients, their adapted response was missing a fundamental characteristic. The control subjects exhibited robust forgetting during each set break: the vertical endpoint of control saccades suddenly decreased (Fig. 5A, first arrow). This set structure was prominent when we plotted the changes in saccade parameters with respect to the last bin (last two saccades) of each set (Fig. 5C). On average, the vertical endpoint declined by $0.43^{\circ}$ or $26 \%$ at set start ( $p<0.005$, set start vs previous set end) 

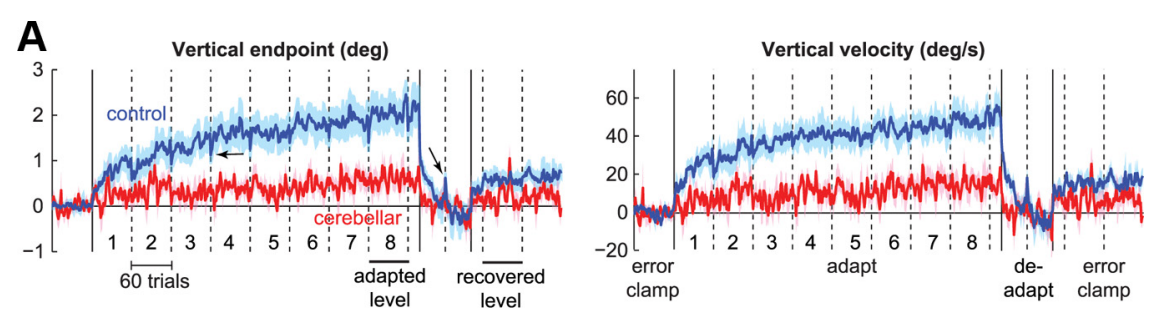

B
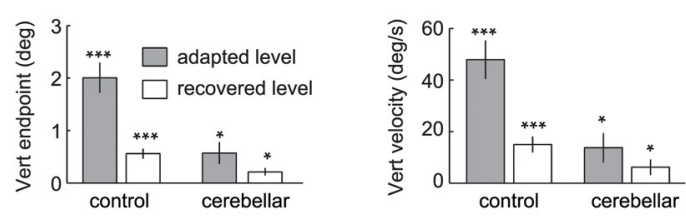

C
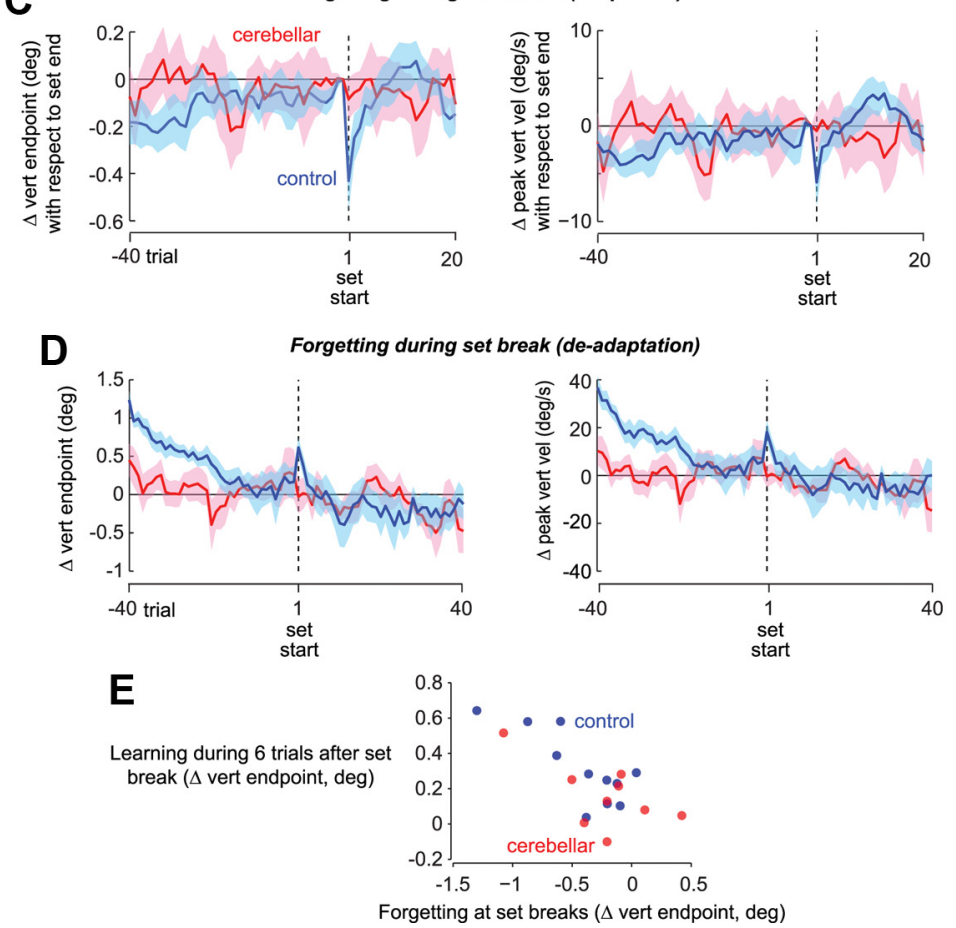

Figure 5. The multiple timescales of adaptation. $\boldsymbol{A}$, The plots show the vertical endpoint of the primary saccade and its peak vertical velocity. Cerebellar patients (red) are impaired in adapting to cross-axis target jumps compared with controls, but nevertheless show significant adaptation. In the deadapt period, the behavior of both groups returned to baseline, but in the following error-clamp trials, there was partial recovery. Dashed vertical lines denote set breaks. Solid vertical lines denote changes of trial types. Note that in the control group, there is forgetting (first arrow) at each set break followed by rapid relearning. Also note that forgetting reverses direction (second arrow) in the deadaptation period. $\boldsymbol{B}$, Summary of the performance at the final set of adaptation and during the first 60 trials of error-clamp Il in controls and patients $\left({ }^{*} p<0.05,{ }^{* *} p<0.01\right.$, ${ }^{* * *} p<0.001$ ). Error bars indicate SEM. C, Vertical endpoint and velocity aligned on set start, as in Figure $4 B$. The forgetting followed by rapid relearning is present in controls but absent in patients. $\boldsymbol{D}$, Vertical endpoint and velocity at the set break in the deadaptation block. Only the controls exhibit reverse-forgetting. Error bars indicate SEM. E, Rapid learning within the first 6 trials of each adaptation set and forgetting at set breaks for individual subjects. The control group showed a strong correlation of $r^{2}=0.62$ with $p<0.004$. The cerebellar subjects showed a marginally significant correlation of $r^{2}=0.40$ with $p=0.067$.

and the peak vertical velocity declined by $5.8 \%$ s or $14.3 \%(p<$ $0.05)$. By the sixth saccade after set start the vertical endpoints and velocities had recovered to the magnitude of the previous set (Fig. 5C). That is, the short break produced forgetting, and the set restart produced rapid relearning. When viewed as a group, both the forgetting and the rapid relearning were absent in the saccades of the cerebellar patients.

When we analyzed the data in terms of individual subjects, we found a strong correlation between a subject's tendency to forget at set breaks and the ability to learn rapidly after set start. For example, Figure $5 E$ shows the change in vertical endpoint position during the first 6 trials after the set start (relearning) as a function of change in the same index during the set break (forgetting). These two measures were strongly correlated in the control subjects $(r=0.79, p<0.004)$ and marginally significant in the cerebellar subjects $(r=$ $0.63, p=0.068$ ). The one cerebellar subject who showed forgetting at set break also showed rapid relearning at set start.

It is possible that the rapid changes in performance after set start are not due to rapid relearning, but a contextual effect in which there is remanifestation of a previously learned state. There is a simple way to check for this. If the forgetting and rapid relearning are both due to a fast adaptive process, then the same fast learning should be present at the very first adaptation set as well as after each set break. On the other hand, if the rapid change after set start was due to revisiting a previous context, then it should be absent in the first set as that context had not been repeated before. In the control group, we found rapid learning in the very first set: the change in vertical velocity by trial 6 was $12.56^{\circ} / \mathrm{s}(p<0.05)$, which was no different from the average change observed after set breaks (within-subject $t$ test, $p=0.33$ ). Similarly, the vertical endpoint changed by $0.34^{\circ}(p<0.05)$ in the first 6 trials of the first set, which was no different from changes seen after set breaks (within-subject $t$ test, $p=0.57$ ). This is consistent with the idea that set breaks induced forgetting, and set restart induced relearning; both of which are signatures of a fast adaptive process.

We were concerned that for the cerebellar subjects, we could not detect forgetting during the set-breaks because they had learned only a small amount. Therefore, we focused on the last three adaptation sets as during these sets the response showed significant adaptation. Despite this, we could not detect a robust change in the cerebellar saccades at set start in our measures of adaptation ( $p=0.13$ for vertical endpoints and $p=0.12$ for vertical velocity).

An interesting prediction of the idea that learning in healthy people is supported by two timescales is that when adaptation is followed by deadaptation, the direction of forgetting should reverse (Ethier et al., 2008). To explain this, consider that during the deadaptation period a competition may be formed between a fast adaptive mechanism that learns the CW perturbation, and the slow adaptive mechanism that previously has learned the CCW perturbation. During the set break in the deadaptation block the fast mechanism should forget, and the behavior should revert to what the slow mechanism had learned. Whereas during the adaptation 
period forgetting during set breaks was toward baseline, now in the deadaptation period forgetting should be away from baseline toward the CCW value stored by the slow system.

Indeed, for the control subjects the set break in the deadaptation period produced "reverse-forgetting" (recovery of $0.45^{\circ}$ in vertical endpoint and $12.3^{\circ}$ s in vertical velocity, $p<0.05$, second arrow in Fig. $5 A$, close-up of the deadapt period in Fig. $5 D$ ), but this pattern was missing in the cerebellar subjects $(p=0.25)$. This result further confirms that the fast timescale of adaptation was present in the control subjects but missing in the patients.

Our simple two-timescale model could not account for one aspect of the data. If the return of performance to baseline (i.e., washout or extinction) during deadaptation was solely due to a competition between two timescales of adaptation, and if cerebellar subjects were impaired in the fast timescale, these subjects should show a slower than normal rate of deadaptation. This was not the case. In the deadaptation period, vertical endpoint and velocity of saccades in cerebellar subjects returned to baseline even faster than controls (Fig. 5A). This possibly indicates that deadaptation in cerebellar subjects benefited from the ability to inhibit a previously learned pattern, rather than set up a competition between a fast and a slow adaptive process.

The deadaptation block was followed by an error-clamp block. In this block, both groups exhibited spontaneous recovery of their previously adapted behavior. In controls, vertical endpoints and velocities were significantly greater than baseline $(p<0.001)$ (Fig. $5 B)$. Similarly, in the cerebellar patients vertical endpoints and velocities were significantly greater in the final error-clamp block than baseline $(p<0.05)$. Spontaneous recovery is a signature of the slow adaptive system that resists "unlearning" during the deadaptation period (Ethier et al., 2008). On average, the magnitude of the spontaneous recovery in controls was $28 \%$ of the state achieved during the adaptation block. In the cerebellar patients, the magnitude of the spontaneous recovery was $33 \%$ of the state achieved during the adaptation block. Importantly, there were no significant differences in the magnitude of percent spontaneous recovery in the two groups.

\section{Saccade curvature}

A prominent feature of saccades in cross-axis adaptation is curvature (Chen-Harris et al., 2008); i.e., motor commands that initiate the saccade appear to adapt by a smaller amount than those that terminate the saccade. A proxy for curvature is the difference in the slopes of the saccade near its start and finish. We divided each saccade into four equal horizontal segments and measured the slope of each segment. Figure $6 \mathrm{~A}$ shows the slope at saccade start (termed S1) and the slope at saccade end (termed S4). In both groups, S1 increased significantly during the adaptation block $\left(+0.12, p<10^{-4}\right.$ in controls, $+0.028, p<0.05$ in cerebellar patients), with the changes being significantly smaller
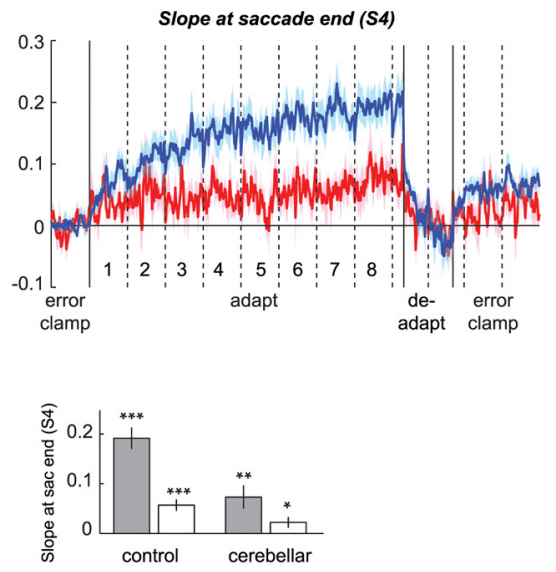

Forgetting during set breaks (adaptation)

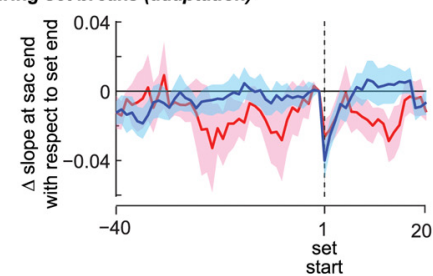

orgetting during set break (de-adaptation)

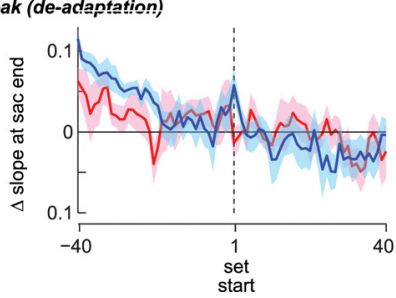

set

Figure 6. Early versus late part of single saccades. We divided each saccade into four equal horizontal segments and measured the slope of each segment. $\boldsymbol{A}$, The slope at saccade start is termed $\mathrm{S} 1$ and the slope at saccade end S4. B, Summary patients $\left({ }^{*} p<0.05,{ }^{* *} p<0.01,{ }^{* * *} p<0.001\right)$. C, Within-subject change in $S 1$ and $S 4$ with respect to the end of the previous set. $\boldsymbol{D}$, Within-subject change at the set break in the deadaptation block. Error bars indicate SEM.

in cerebellar patients $(p<0.001)$. In both groups, S4 was larger than $S 1$, resulting in saccades that curved toward the target (within group comparison, last set of adaptation, $+0.19, p<$ $10^{-5}$ in controls, $+0.073, p<0.01$, in cerebellar patients). When the trial-to-trial changes in slope were aligned on set starts, we once again observed the fast timescales of learning in the healthy controls (S1 dropped by 0.039 or $35 \%, p=0.01$, S4 dropped by 0.041 or $26 \%, p=0.01$ ), but not clearly in the cerebellar group: $\mathrm{S} 1$ showed no drop at set break ( $p=0.3$ ), and while $S 4$ dropped by $0.026,42 \%$ at set break, it showed similar drops both before the set break and after the set break. Similarly, Figure $6 D$ illustrates that the set-break during deadaptation produced reverseforgetting in the control subjects (recovery of 0.34 for $\mathrm{S} 1, p<$ 0.05 , recovery of 0.028 for $S 4, p<0.05$ ), but not in the cerebellar group ( $p=0.4$ for $\mathrm{S} 1$ and $p=0.15$ for $\mathrm{S} 4$ ).

\section{Ability to compensate for variability predicts ability to learn from endpoint errors}

Finally, we asked whether a subject's ability to control endpoint accuracy during the preadaptation control trials was a predictor of their ability to learn from endpoint errors during adaptation trials. Our proxy for the ability to control accuracy was endpoint variability (SD) and endpoint bias in the oblique control trials, before the adaptation trials began. Our proxy for the ability to 

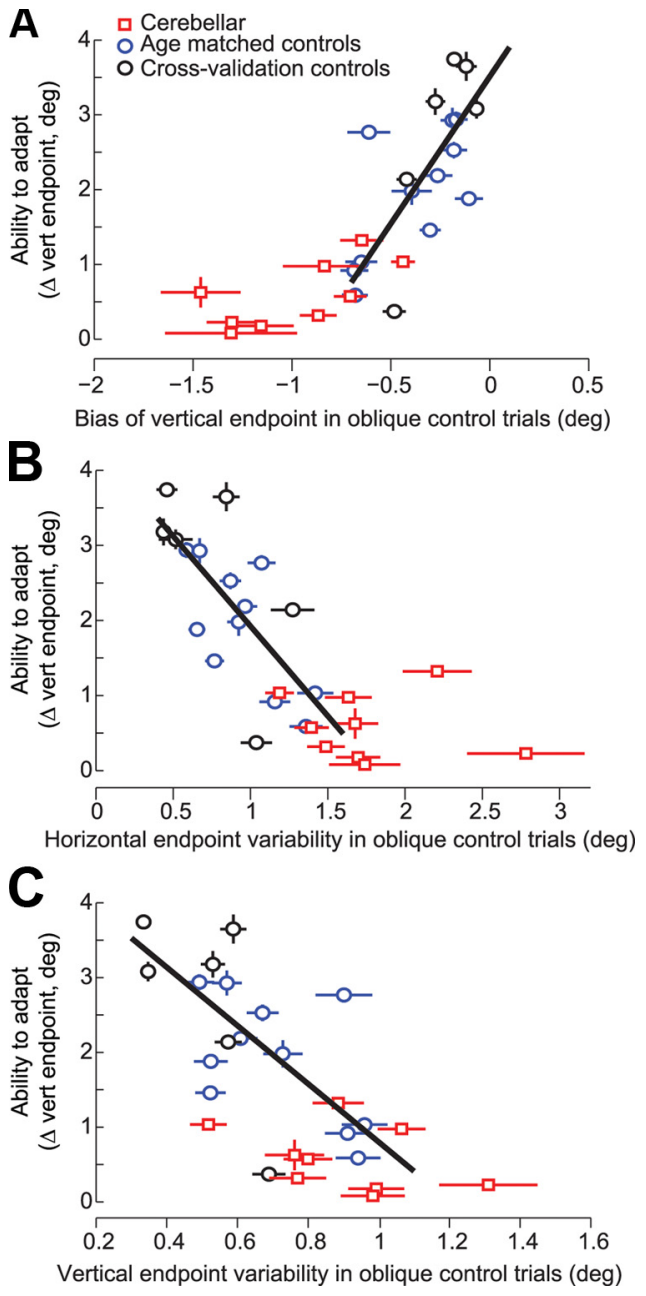

Figure 7. The ability to compensate for internal sources of variability correlates with the ability to compensate for external sources of error. $\boldsymbol{A}$, Correlation between bias in the vertical direction for oblique trials and learning along the vertical direction during adaptation. The best fit line is for control subjects. Error bars are SEM. B, C, Horizontal (or vertical) endpoint variability before adaptation is plotted on the $x$-axis and the ability to adapt to endpoint errors during adaptation trials is plotted on the $y$-axis. The best fit line is for all control subjects. Error bars for horizontal and vertical variability are SEs of the SD estimate.

adapt was the vertical endpoint achieved in the final set of the adaptation block. As a group, the cerebellar patients had larger vertical bias (negative value means undershooting, $p<0.001$ ) (Fig. 7A), more horizontal endpoint variability $(p<0.01)$ (Fig. $7 B)$, and more vertical endpoint variability $(p<0.05)$ (Fig. $7 C$ ) than controls. Inclusion of the cerebellar group with healthy controls in a regression analysis would, of course, produce a significant correlation between control of saccade accuracy and adaptation. However, a more interesting question is whether within the control subjects, the ability to control endpoint variability was a predictor of the ability to adapt. Indeed, in healthy subjects the ability to control saccades during the oblique trials was a predictor of the ability to adapt to errors during the adaptation trials (blue circles, Fig. 7A-C, vertical bias: $r^{2}=0.43, p<0.05$; horizontal variability: $r^{2}=0.50, p<0.05$; vertical variability was close to significance: $r^{2}=0.32, p=0.06$ ). (In comparison, we found no correlation between horizontal bias and amount of learning.)

To test the strength of the correlation between endpoint variability and learning, we considered a cross-validation procedure. We added to our analysis six additional control subjects (Fig. 7, black circles) who were not age-matched to the patients. Within this larger control group, we found an even stronger relationship between the trial to trial control of saccade accuracy and vertical adaptation (vertical bias: $r^{2}=0.51, p<0.005$; vertical variability: $r^{2}=0.42, p<0.005$; horizontal variability: $\left.r^{2}=0.50, p=0.001\right)$.

To further test the idea that the ability to control endpoint variability predicts the ability to adapt, we considered other measures of within-trial saccadic control. For example, as saccade horizontal velocity decreases, durations should increase to maintain horizontal amplitude. This implies that for a given subject, a negative correlation between peak horizontal velocity and duration is indicative of better endpoint control. Indeed, this measure was also a predictor of the ability to learn from endpoint errors: the more negative the correlation between peak horizontal velocity and duration, the better the ability of that subject to learn from vertical endpoint errors $\left(r^{2}=0.27, p<0.05\right.$ for all controls $)$. In another example of within-saccade control, consider that as saccade horizontal velocity changes, the more positive the correlation with horizontal amplitude, the less perfect the compensation (e.g., if a decrease in velocity is not compensated, the result is a decrease in amplitude). Indeed, people who exhibited a positive correlation between horizontal velocity and amplitude were generally the subjects who also learned the least from the vertical endpoint errors ( $r^{2}=0.30, p<0.05$ for all controls).

In summary, we found that a healthy subject's ability to control endpoint accuracy during the preadaptation control trials predicted their ability to learn from endpoint errors during adaptation trials.

\section{Discussion}

Some three decades ago, David Robinson proposed that saccadic motor commands are monitored and corrected to steer the eyes to the target (Robinson, 1975). Later work suggested the cerebellum was central to this monitoring process (Quaia et al., 1999). In the current computational view of motor control (Shadmehr and Krakauer, 2008) the cerebellum may be a forward model (Pasalar et al., 2006) that uses efferent copy to predict consequences of motor commands and contributes to the online correction of movement. Presumably, the forward model learns from endpoint errors to maintain accuracy. Saccades (compared with reaching movements) are particularly useful for testing the theory of forward models because these eye movements are completed too quickly for visual or proprioceptive feedback to play a role in control. Here, we wished to quantify the influence of the cerebellum on both the within-saccade compensation of the commands that initiated the movement, and the longer-term learning that compensated for persistent errors.

Our subjects were healthy people and patients with a neurodegenerative disorder (SCA-6) that affected the Purkinje cells of the cerebellum. As noted before (Chen-Harris et al., 2008), repetition of a target on the horizontal meridian gradually reduced horizontal saccade velocities, and short breaks rapidly increased these velocities. In healthy people, this variability was generally compensated via corrective motor commands that arrived later in the same saccade. However, the compensation was missing in cerebellar subjects. In healthy people, the within-saccade ability to compensate for the variability in the horizontal velocity was a predictor of the ability to adapt to errors in the vertical direction. The adaptation relied on a memory that exhibited multiple timescales: a fast process that learned quickly from endpoint errors but showed forgetting during the short set breaks, and a slow process that learned gradually, resisted unlearning, and became latent during a brief period of deadaptation but reemerged after cessation of the deadapting stimulus. Despite significant adaptation in the cerebellar subjects, their adapted saccades were miss- 
ing the forgetting and the rapid-relearning, suggesting that the damage to the cerebellar cortex had produced a deficit that mostly affected the fast timescale of adaptation.

\section{A source of internal variability in the motor commands that initiate a saccade}

In this study, only healthy controls were able to compensate for variability due to repetition (gradual decline of saccade horizontal velocity and its rapid recovery after set breaks). This compensation was via a change in the time course of the deceleration phase, late in the saccade. Therefore, while the source of the variability appeared to be outside the cerebellar cortex (as it was present in both the patients and the controls), only subjects with a healthy cerebellum corrected for the variability.

Saccade slowing could be due to fatigue of the oculomotor plant or cognitive factors. Here, we gained new insights into the source of this repetition attenuation of saccade velocities: we found that an unexpected change in the repeating target produced immediate recovery of the horizontal velocities. This suggests that the slowing of the saccades and its recovery at set breaks were unrelated to neuromuscular fatigue in the oculomotor plant, as recovery would require passage of time. Sensory neurons that encode some particular attribute of a stimulus usually show progressively smaller responses when that attribute is repeated (Miller and Desimone, 1994; Kohn and Movshon, 2003). This "repetition suppression" has been observed in area V1 of the occipital cortex (Grill-Spector et al., 2006) and in the superficial layer of the superior colliculus (Boehnke et al., 2007). Because the receptive field of these cells is retinotopic and the unexpected change in the repeating stimuli did not alter its positions in retinotopic coordinates, the recovery of velocity cannot be explained by recruitment of new sensory neurons with different receptive fields. That is, the repetition attenuation is probably not a "bottom-up" phenomenon. However, it is known that the brain directs attention to a visual stimulus that has behaved differently than expected (Itti and Baldi, 2009). It seems likely that the repetition attenuation in saccade velocities was due to top-down cognitive factors like attention.

Our observation agrees well with the data from Golla et al. (2008) who also noted a drop in saccade velocities that went uncompensated in cerebellar patients. A possible explanation is that the cerebellum receives a copy of the motor commands and then makes adjustments to steer the eyes to the target (Quaia et al., 1999). Indeed, cells in the cerebellar vermis and caudal fastigial nucleus show discharges with timing that could be used to adjust motor commands during the deceleration phase of the saccade (Ohtsuka and Noda, 1992; Fuchs et al., 1993; Catz et al., 2008).

\section{The multiple timescales of adaptation}

In healthy people, adaptation appeared to consist of two distinct processes: a fast process that exhibited forgetting and relearning at set breaks, and a slow process that exhibited little forgetting and strong resistance to unlearning when the endpoint errors suddenly reversed direction (the deadaptation period). We had earlier observed a similar " two-state" process in a gain adaptation paradigm (Ethier et al., 2008), and in a reaching paradigm (Smith et al., 2006; Criscimagna-Hemminger and Shadmehr, 2008). In cerebellar patients, the fast adaptation process appeared to be impaired as the learned vertical component did not exhibit forgetting during rest periods. In contrast, the slow process was at least partially spared in the patients as the learned vertical component exhibited recovery during the error clamp period that followed the brief deadaptation stimulus.
Is the lack of evidence for a fast adaptive process in cerebellar patients due to increased noise in these subjects? Indeed, it is possible that our inability to observe a component of adaptation in our subject group was due to sample size and increased variability in that group. However, note that we found significant changes in horizontal velocities in the saccades of the same patients at set breaks, but no forgetting in the vertical velocities (Fig. 4C). Changes in horizontal velocities are unrelated to adaptation (Chen-Harris et al., 2008), and are examples of fast cognitive or attentional processes that affected saccades of both patients and healthy controls.

There are experimental results that support the idea that the cerebellar cortex may be specialized for fast processes that underlie motor memory, whereas the deep cerebellar nuclei may specialize in the slow processes. For example, in classical conditioning of the eye blink reflex, washout of the previous learning still results in a more rapid reacquisition than when the subject is naive, a phenomenon termed savings. Medina et al. (2001) have attributed this to different timescales of learning in the cerebellar cortex and nuclei. Barash et al. (1999) suggested that there are two processes that adjust the saccade gain (eye displacement/target amplitude): the rapid gain adjustment mechanism depends on the cerebellar cortex while the slower adjustment takes place outside of the cerebellar cortex. Similar suggestions have been made for the role of the cerebellum in other types of oculomotor learning (Ohyama et al., 2006; Shutoh et al., 2006; Masuda and Amari, 2008).

\section{Are the changes in the vertical motor commands an adaptive response related to deficits in the horizontal motor commands?} At set starts, we found that the internal/cognitive perturbation is effectively absent since horizontal saccade velocity recovered. At the same time, vertical motor commands showed a large reduction in control subjects. That is, when there was little need to correct for deficits in the motor commands that initiated the saccade, there was also a reduced ability to express learning, i.e., an apparent forgetting. The cerebellar subjects, however, displayed neither the ability to correct for changes in the horizontal motor commands nor the forgetting. One hypothesis to explain this observation is that the cerebellum learns a compensatory response in the vertical direction, but this response can only be expressed when there is a deficit in the horizontal motor commands that initiate the saccade. In this view, the term "forgetting" is unjustified because the changes in set breaks are not due to decay or loss of a previously learned response, but simply a lack of necessity to express it.

Although this is an attractive hypothesis, there are a number of patterns in our data that are inconsistent with it. First, the rapid learning that follows set breaks has a much faster rate (achieving previously learned level within 6 trials) than the repetitioninduced drop in the horizontal velocity, which slows over the course of many more trials. That is, changes in the horizontal velocities alone could not explain the forgetting and rapid relearning in the vertical direction. Second, at set start we found robust reductions in not just the vertical commands that terminated the saccade, but also the vertical commands that initiated it (Fig. 6C). Therefore, forgetting in the vertical motor commands occurred too early into a saccade to have been a response to changes in horizontal motor commands. Finally, if set breaks did not produce forgetting, then the rapid relearning after set start is a remanifestation of a previously learned state, which should not be present in the first adaptation set. In fact, rapid learning was present in the first adaptation set. Together, the data are more consistent with the idea that the time passage during set breaks induced forgetting, the large errors at set restart induced relearning, and damage to the cerebellar cortex primarily influenced these fast timescales of adaptation. 


\section{The ability to compensate for internal sources of variability correlates with the ability to compensate for external sources of error}

In our experiment, there were two potential sources of movement error: one due to internal variability in the motor commands that initiated the saccade, and the other due to external perturbations of the target. We found that a subject's ability to control endpoint accuracy during the preadaptation control trials predicted their ability to learn from endpoint errors during adaptation. The relationship is consistent with other studies (van der Geest et al., 2006), suggesting that maintenance of saccade accuracy in daily life and short-term saccade adaptation have shared neural mechanisms, perhaps dependent on the cerebellum (Takagi et al., 1998; Barash et al., 1999). Alternatively, greater endpoint variability causes lack of adaptation by providing inconsistent errors to drive learning. It is also possible that subjects with greater inherent variability attribute more error to their own motor output, which does not warrant updating of the internal representation of the visual environment (Burge et al., 2008; Wei and Körding, 2009). If increased variability indeed causes lack of adaptation, one could test this idea by inducing adaptation by clamping the error with respect to the saccade endpoint. This way control subjects and patients would perceive the same motor performance and receive the same error information.

In summary, we found that there is variability in motor commands that accelerate the eyes, but this variability is partially corrected within a saccade via the cerebellum. In healthy people, the ability to compensate for variability is correlated with the ability to learn from endpoint errors. While damage to the cerebellar cortex significantly impairs the ability to learn from endpoint errors, the impairment is focused on a fast adaptive process that learns rapidly from error but also exhibits significant forgetting with passage of time.

\section{References}

Barash S, Melikyan A, Sivakov A, Zhang M, Glickstein M, Thier P (1999) Saccadic dysmetria and adaptation after lesions of the cerebellar cortex. J Neurosci 19:10931-10939.

Boehnke SE, Berg DJ, Marino RA, Itti L, Munoz DP (2007) Adaptation, habituation and dishabituation of visual responses in the superior colliculus. Soc Neurosci Abs 617.12/PP14.

Burge J, Ernst MO, Banks MS (2008) The statistical determinants of adaptation rate in human reaching. J Vis 8:1-19.

Catz N, Dicke PW, Thier P (2008) Cerebellar-dependent motor learning is based on pruning a Purkinje cell population response. Proc Natl Acad Sci U S A 105:7309-7314.

Chen-Harris H, Joiner WM, Ethier V, Zee DS, Shadmehr R (2008) Adaptive control of saccades via internal feedback. J Neurosci 28:2804-2813.

Criscimagna-Hemminger SE, Shadmehr R (2008) Consolidation patterns of human motor memory. J Neurosci 28:9610-9618.

Ethier V, Zee DS, Shadmehr R (2008) Spontaneous recovery of motor memory during saccade adaptation. J Neurophysiol 99:2577-2583.

Fuchs AF, Robinson FR, Straube A (1993) Role of the caudal fastigial nucleus in saccade generation. I. Neuronal discharge pattern. J Neurophysiol 70:1723-1740.

Golla H, Tziridis K, Haarmeier T, Catz N, Barash S, Thier P (2008) Reduced saccadic resilience and impaired saccadic adaptation due to cerebellar disease. Eur J Neurosci 27:132-144.

Grill-Spector K, Henson R, Martin A (2006) Repetition and the brain: neural models of stimulus-specific effects. Trends Cogn Sci 10:14-23.

Honjo K, Ohshita T, Kawakami H, Naka H, Imon Y, Maruyama H, Mimori Y, Matsumoto M (2004) Quantitative assessment of cerebral blood flow in genetically confirmed spinocerebellar ataxia type 6. Arch Neurol 61:933-937.

Ishikawa K, Watanabe M, Yoshizawa K, Fujita T, Iwamoto H, Yoshizawa T, Harada K, Nakamagoe K, Komatsuzaki Y, Satoh A, Doi M, Ogata T, Kanazawa I, Shoji S, Mizusawa H (1999) Clinical, neuropathological, and molecular study in two families with spinocerebellar ataxia type 6á(SCA6). J Neurol Neurosurg Psychiatry 67:86-89.
Itti L, Baldi P (2009) Bayesian surprise attracts human attention. Vision Res 49:1295-1306.

Koeppen AH (2005) The pathogenesis of spinocerebellar ataxia. The Cerebellum 4:62-73.

Kohn A, Movshon JA (2003) Neuronal adaptation to visual motion in area MT of the macaque. Neuron 39:681-691.

Kojima Y, Iwamoto Y, Yoshida K (2004) Memory of learning facilitates saccadic adaptation in the monkey. J Neurosci 24:7531-7539.

Kording KP, Tenenbaum JB, Shadmehr R (2007) The dynamics of memory as a consequence of optimal adaptation to a changing body. Nat Neurosci 10:779-786.

Masuda N, Amari S (2008) A computational study of synaptic mechanisms of partial memory transfer in cerebellar vestibulo-ocular-reflex learning. J Comput Neurosci 24:137-156.

Matsuyama Z, Kawakami H, Maruyama H, Izumi Y, Komure O, Udaka F, Kameyama M, Nishio T, Kuroda Y, Nishimura M, Nakamura S (1997) Molecular features of the CAG repeats of spinocerebellar ataxia 6 (SCA6). Hum Mol Genet 6:1283-1287.

Medina JF, Garcia KS, Mauk MD (2001) A mechanism for savings in the cerebellum. J Neurosci 21:4081-4089.

Miller EK, Desimone R (1994) Parallel neuronal mechanisms for shortterm memory. Science 263:520-522.

Ohtsuka K, Noda H (1992) Burst discharges of mossy fibers in the oculomotor vermis of macaque monkeys during saccadic eye movements. Neurosci Res 15:102-114.

Ohyama T, Nores WL, Medina JF, Riusech FA, Mauk MD (2006) Learninginduced plasticity in deep cerebellar nucleus. J Neurosci 26:12656-12663.

Optican LM, Robinson DA (1980) Cerebellar-dependent adaptive control of primate saccadic system. J Neurophysiol 44:1058-1076.

Pasalar S, Roitman AV, Durfee WK, Ebner TJ (2006) Force field effects on cerebellar Purkinje cell discharge with implications for internal models. Nat Neurosci 9:1404-1411.

Quaia C, Lefèvre P, Optican LM (1999) Model of the control of saccades by superior colliculus and cerebellum. J Neurophysiol 82:999-1018.

Robinson DA (1963) A method of measuring eye movement using a scleral search coil in a magnetic field. IEEE Trans Biomed Eng 10:137-145.

Robinson DA (1975) Oculomotor control signals. In: Basic mechanisms of ocular motility and their clinical implications (BachyRita P, Lennerstrand G, eds), pp 337-374. Oxford, UK: Pergamon.

Sasaki H, Kojima H, Yabe I, Tashiro K, Hamada T, Sawa H, Hiraga H, Nagashima K (1998) Neuropathological and molecular studies of spinocerebellar ataxia type 6 (SCA6). Acta Neuropathol 95:199-204.

Shadmehr R, Krakauer JW (2008) A computational neuroanatomy for motor control. Exp Brain Res 185:359-381.

Shutoh F, Ohki M, Kitazawa H, Itohara S, Nagao S (2006) Memory trace of motor learning shifts transsynaptically from cerebellar cortex to nuclei for consolidation. Neuroscience 139:767-777.

Smith MA, Ghazizadeh A, Shadmehr R (2006) Interacting adaptive processes with different timescales underlie short-term motor learning. PLoS Biol 4:e179.

Snyder LH, Calton JL, Dickinson AR, Lawrence BM (2002) Eye-hand coordination: saccades are faster when accompanied by a coordinated arm movement. J Neurophysiol 87:2279-2286.

Straube A, Fuchs AF, Usher S, Robinson FR (1997) Characteristics of saccadic gain adaptation in rhesus macaques. J Neurophysiol 77:874-895.

Straube A, Deubel H, Ditterich J, Eggert T (2001) Cerebellar lesions impair rapid saccade amplitude adaptation. Neurology 57:2105-2108.

Takagi M, Zee DS, Tamargo RJ (1998) Effects of lesions of the oculomotor vermis on eye movements in primate: saccades. J Neurophysiol 80:1911-1931.

Takikawa Y, Kawagoe R, Itoh H, Nakahara H, Hikosaka O (2002) Modulation of saccadic eye movements by predicted reward outcome. Exp Brain Res 142:284-291.

van der Geest JN, Haselen GC, Frens MA (2006) Saccade Adaptation in Williams-Beuren Syndrome. Invest Ophthalmol Vis Sci 47:1464-1468.

van Donkelaar P (1997) Eye-hand interactions during goal-directed pointing movements. Neuroreport 8:2139-2142.

Wei K, Körding K (2009) Relevance of Error: What Drives Motor Adaptation? J Neurophysiol 101:655-664.

Xu-Wilson M, Zee DS, Shadmehr R (2009) The intrinsic value of visual information affects saccade velocities. Exp Brain Res 196:475-481.

Zee DS, Yee RD, Cogan DG, Robinson DA, Engel WK (1976) Ocular motor abnormalities in hereditary cerebellar ataxia. Brain 99:207-234. 